\title{
The William Harvey Research Institute The Barts Heart Centre, Queen Mary University of London, UK
}

\section{Mark Caulfield}

\section{The William Harvey Research Institute - Barts heritage}

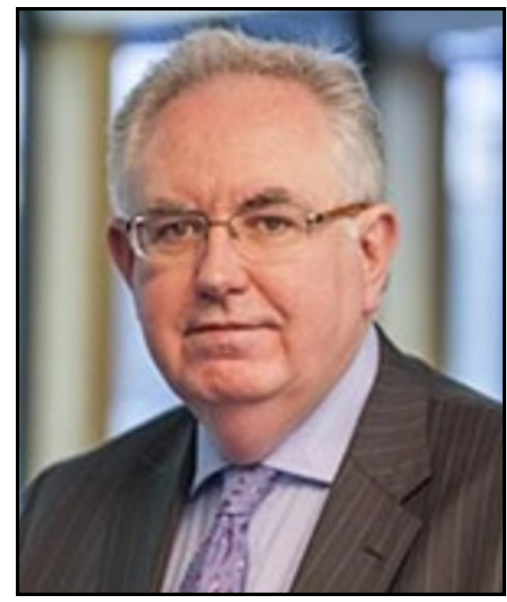

Our hospital, St Bartholomew's Hospital (Barts), has been caring for the people of London since 1123 and whilst a Physician at Barts, in 1628 William Harvey published de motu cordis, providing the modern view of the circulation. In 1986 the Nobel Laureate, Sir John Vane FRS, who discovered how aspirin worked, identified prostacyclin and held a pivotal role in the development of ACE inhibitors founded the William Harvey Research Institute (WHRI) at Barts and Queen Mary (OMUL). Today, the WHRI is home to 520 clinicians and scientists and $90 \%$ of their translational research in cardiovascular (CV), inflammation and endocrine research was independently rated as internationally excellent, or world-leading in 2014. In 2006, the strength of this WHRI-OMUL and Barts partnership led to recognition as a Hypertension Centre of Excellence of the European Society of Hypertension (ESH). It benefits greatly from being part of QMUL a 400 million turnover multi-faculty university and member of the Russell Group of 24 elite UK Universities.

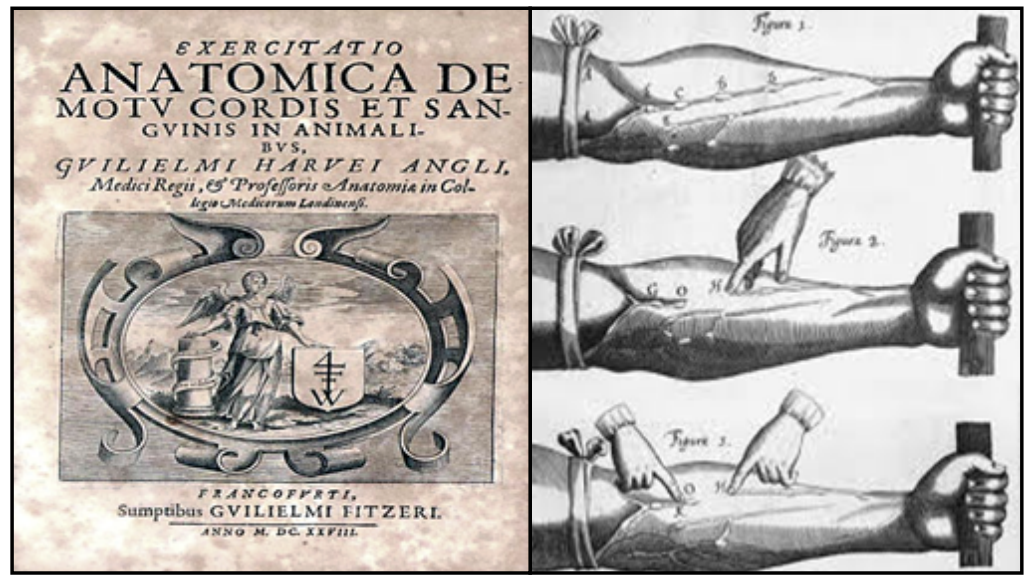

William Harvey's de Motu Cordis and his illustration of valves in veins published in 1628 when he was Physician to St Bartholomew's Hospital

\section{State of the art infrastructure at WHRI-OMUL and the Barts Heart Centre}

In 2011, we opened the William Harvey Heart Centre which provides a state-of-the art CV clinical research centre and translational laboratories. In parallel, a five-year programme co-led by the co-Director of WHRI, Prof Mark Caulfield united three hospitals at the $£ 400$ million Barts Hospital creating the new Barts Heart Centre in 2015. The Barts Heart Centre is one of the largest cardiovascular centres in Europe providing 80,000 patient episodes of care per year and serving a population of six million across North East London. This combination led to the award of a prestigious National Institute for Health Research Biomedical Research Centre to Barts in successive competitions since 2008. 


\section{Translational Blood Pressure Research at the William Harvey-QMUL and Barts.}

A major research focus at WHRI-OMUL is on blood pressure (BP) and Caulfield has driven a world-leading integrated programme tackling unmet need in hypertension care. This operates under a series of interconnected themes taking discoveries from genomics into target validation and therapeutic innovation which is translated into patient care at the Barts Heart Centre.

The $\mathbf{4 0 0}$ million new Barts Heart Centre and the WHRI integrated strategy for therapeutic innovation in hypertension and CV prevention.

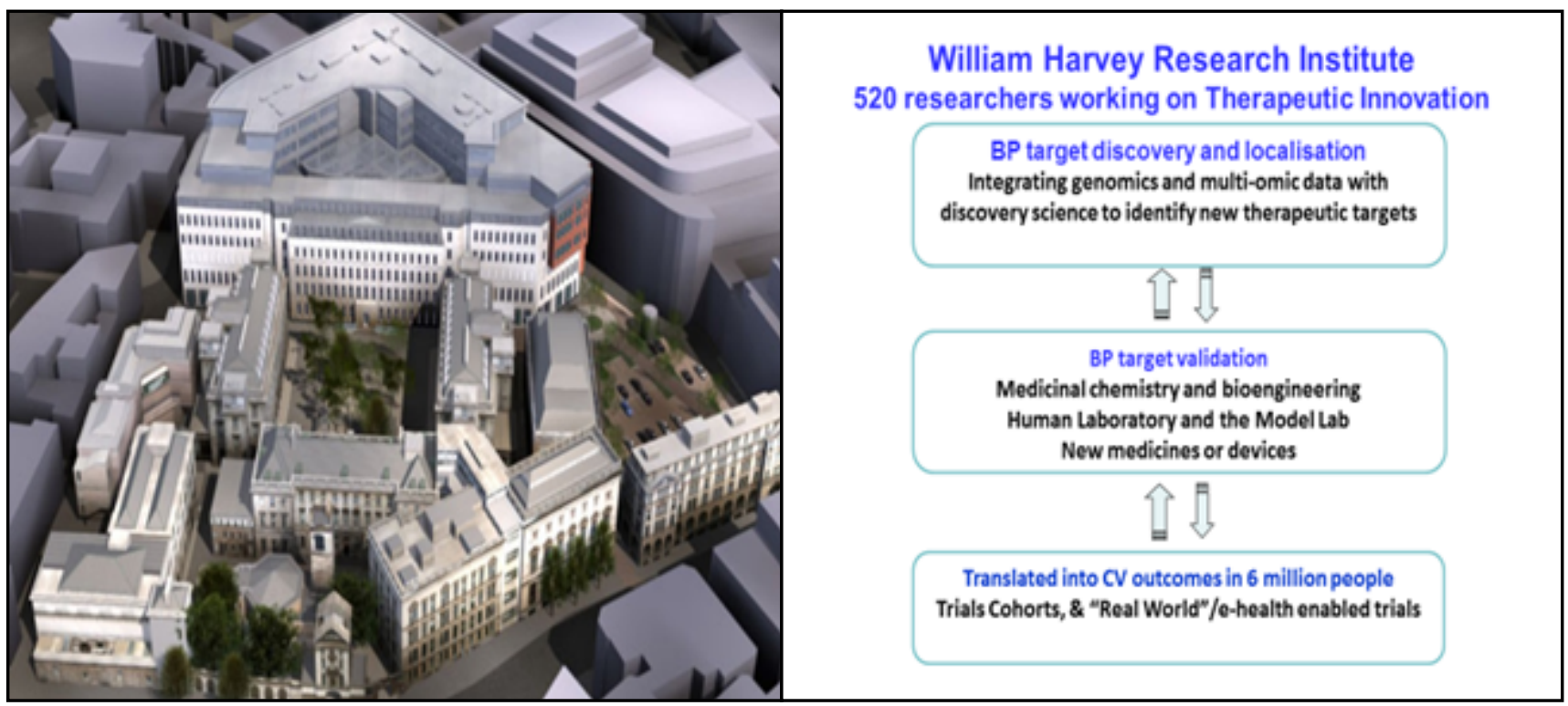

Genomics of BP and CV disorders (Profs. Caulfield FMedSci, Patricia Munroe, Morris Brown FMedSci, Deloukas FMedSci, Drs Fu Ng, Helen Warren, Claudia Cabrera, Mike Barnes): Our genomics programme has now identified over 1000 loci for blood pressure traits, demonstrating in a polygenic risk score that these loci lead to a $13 \mathrm{~mm} \mathrm{Hg}$ systolic BP in the over 50 year olds and correlate with increased CV risk 1. From the leadership of the MRC British Genetics of Hypertension Study and the hypertension theme in the 2007 Nature paper from the Wellcome Trust Case Control Consortium we formed the International Consortium of Blood Pressure Genomewide Studies leading to a succession of publications in Nature or Nature Genetics. Our most recent study of over one million people, including 500,000 from the UK Biobank cohort, has identified 535 new loci for blood pressure connected to multiple extant and putative medications for hypertension ${ }^{1}$. Through our leadership of the MRC-funded International Mouse Phenotyping Consortium CV programme we can create the latest gene edited murine models for functional studies and combine these in our human laboratory to undertake ex-vivo human vascular and renal cell based studies as we have done for the Natriuretic Peptide C Receptor (NPR3), SLC4A9 and SLC39A8 genes.

Endocrine Hypertension (Profs Morris Brown, William Drake, Paul Chapple): In 2016 Brown joined us from Cambridge to pursue, with Drake, research into pathogenesis and management of primary aldosteronism. The discovery of somatic driver mutations, in aldosterone-producing adenomas of distinctive clinical, pathological and biochemical profile, led to recognition of a frequently overlooked zona glomerulosa sub-type of adenoma. Their frequency and importance were confirmed by development of a 11C-Metomidate PET CT; this allows non-invasive detection of microadenomas and has enabled ongoing evaluation of endoscopic radiofrequency ablation for adenomas in the left adrenal $^{2}$. The somatic mutations also offered targets for new treatments, now in development, which may suppress autonomous aldosterone secretion. Similar genotype-phenotype research in phaeochromocytoma, by Chapple and Drake, led to the discovery and mechanism of primary cilia loss in phaeochromocytomas whose genotype causes pseudohypoxia or malignancy. 
Vascular and Clinical Pharmacology of BP toward guidelines and global CV prevention (Profs Amrita Ahluwalia, Brown, Hobbs, Caulfield, Drs Collier, Kapil (ISH Austin Doyle Prize 2012), Gupta): Our genomic discoveries highlight the Nitric Oxide-Natriuretic peptide signalling system by integrating this with physiology and leading edge pharmacology Ahluwalia and Kapil have published highly cited papers describing how inorganic nitrate, contained within beetroot juice, can be used to sustainably lower BP in both normal volunteers and hypertensive patients, which is now being investigated in left ventricular hypertension ${ }^{3}$. Hobbs has demonstrated that endothelial specific knockout of C-Natriuretic Peptide (CNP) in mice leads to higher BP and that the CNP mimetic he has synthesised lowers BP and is now being optimised with venture funding as a novel therapy for BP and CV prevention.

From our clinical research centre we were the largest single enrolling centre for the Anglo-Scandinavian Cardiac Outcomes Trial (ASCOT -Collier, Caulfield -1157 patients) which showed lipid lowering prevented CV events and that BP lowering with calcium antagonists and ACE inhibitors was superior to older therapies ${ }^{4}$. This paved the way for adoption of Brown's age based stratification for BP lowering therapy where younger people receive renin angiotensin system blockade and those over 55 years receive a CCB or a diuretic which changed the UK National Institute for Clinical and Healthcare Excellence and British Hypertension Society Guidelines in 2006 and 2011 (Caulfield was on the guideline group). More recently Gupta has been able to analyse data from UK participants in the ASCOT study at 10 years showing that a regimen based on CCBs plus ACE inhibitor is superior at $\mathrm{CV}$ reduction 15 years after the end of the lipid lowering limb and that statin therapy is associated with the "nocebo effect" 5

In the British and Irish Hypertension Society and BHF PATHWAY suite of studies Brown tested whether aggressive early treatment of raised blood pressure could prevent subsequent treatment resistance (PATHWAY 1), is resistant hypertension due to $\mathrm{Na}+$ retention and is spironolactone superior to other drugs (PATHWAY 2), and are $\mathrm{K}+$ sparing diuretics neutral or beneficial in their effect on glucose tolerance (PATHWAY 3). The PATHWAY 2 trial has led the latest ESH/European Society of Cardiology Guidelines to recommend spironolactone as first line for resistant hypertension ${ }^{6}$.

Device based innovation and autonomic function (Prof Melvin Lobo, Dr Manish Saxena). In response to the growing number of national and international referrals of patients with complex hypertension, resistant to conventional drug therapy we embarked upon a leading edge trials programme to investigate the use of device (interventional) therapy of hypertension. We are now a global leader in trial recruitment into studies of device-based therapy of hypertension attracting and participating in design of translational Phase I and II studies (from European and US device manufacturers) with international recognition for the trial outcomes. These include the Symplicity HTN-2 study of radiofrequency renal denervation, the ROX CONTROL HTN study which demonstrated the BP lowering effect of a central iliac arteriovenous anastomosis and the RADIANCE-HTN SOLO randomised proof of concept trial demonstrating the efficacy of a novel ultrasound platform for endovascular renal denervation. Lobo and Kapil have recently secured grant funding to initiate an investigator-led national registry for baroreflex activation therapy (for which Barts will be the sole centre) which will evaluate the safety and efficacy of this therapy in previously untested patient groups including those with afferent baroreflex failure. For complex patients and those being recruited into device therapy of hypertension studies, baseline and post-treatment autonomic function testing is undertaken in the human autonomic function lab, one of only two such units in the whole UK. 
The Barts BP Clinic (Profs Lobo, Caulfield, Drs Kapil, Rull, Ng, Robinson, Abrams). This tertiary translational clinic provides a CV prevention service to national and international referrals of patients whose BP is very challenging to manage including medication intolerance and resistant hypertension. This and our extended partnership with primary care practices across London enables us to connect our translational research from the bench to the bedside via accredited hypertension specialists of the British and Irish Hypertension Society and the ESH.

International partnerships: We have many international collaborations with researchers across the world and a suite of focused strategic partnerships with Yale and Harvard University in the US and the People's Liberation Army Hospital in Beijing.

WHRI and Barts Public and Patient Involvement: (Collier): Our patients and the public are at the heart of everything we do and they are involved directly in setting research agendas and enthusing patients to join Clinical Trials through an initiative called Trials Connect and every year they organise a Science Festival for 400 school children.

Mark Caulfield

m.j.caulfield@qmul.ac.uk

\section{REFERENCES}

1. Evangelou E E Warren HR*, then multiple co-authors then Barnes MR*, Wain LV*, Elliott P* $\neq$, Caulfield MJ*‡

Genetic analysis of over one million people identifies 535 new loci associated with blood pressure traits. Nature Genetics 2018. DOI: $10.1038 / \mathrm{s} 41588-018-0205-\mathrm{x}$

2. Kapil V, Khambata RS, Robertson A, Caulfield MJ, Ahluwalia A. Dietary nitrate provides sustained blood pressure lowering in hypertensive patients: a randomized, phase 2, double-blind, placebo-controlled study. Hypertension. 2015 Feb;65(2):320-7. doi: 10.1161/HYPERTENSIONAHA.114.04675.

3. Dahlof $B$, co-authors then Caulfield $M$, et al. Prevention of cardiovascular events with an antihypertensive regimen of amlodipine adding perindopril as required versus atenolol adding bendroflumethiazide as required, in the Anglo-Scandinavian Cardiac Outcomes Trial-Blood Pressure Lowering Arm (ASCOT-BPLA): a multicentre randomized controlled trial. Lancet. 2005 Sep 10-16;366(9489):895-906. DOI: 10.1016/S0140-6736(05)67185-1

4. Williams B, Brown M]; British Hypertension Society's PATHWAY Studies Group.. Spironolactone versus placebo, bisoprolol, and doxazosin to determine the optimal treatment for drug-resistant hypertension (PATHWAY-2): a randomised, double-blind, crossover trial. Lancet. 2015 Nov 21;386(10008):2059-68. doi:

10.1016/S0140-6736(15)00257-3.

5. Gupta $A$, et al. Long-term mortality after blood pressure-lowering and lipid-lowering treatment in patients with hypertension in the Anglo-Scandinavian Cardiac Outcomes Trial (ASCOT) Legacy study: 16-year follow-up results of a randomised factorial trial. Lancet. 2018 Aug 24. pii: S0140-6736(18)31776-8. doi: 10.1016/S0140-6736(18)31776-8.

6. Lobo, M. D., Sobotka, P. A., Stanton, A., \& Cockcroft, J. R. (2015). Central arteriovenous anastomosis for the treatment of patients with uncontrolled hypertension (the ROX CONTROL HTN study): a randomised controlled trial. The Lancet, 385(9978), 1634-1641. DOI: 10.1016/S0140-6736(14)62053-5 